\title{
Smoked Marijuana as a Cause of Lung Injury
}

\author{
D.P. Tashkin
}

ABSTRACT: Smoked Marijuana as a Cause of Lung Injury. D.P. Tashkin.

In many societies, marijuana is the second most commonly smoked substance after tobacco. While $\Delta^{9}$-tetrahydrocannabinol (THC) is unique to marijuana and nicotine to tobacco, the smoke of marijuana, like that of tobacco, consists of a toxic mixture of gases and particulates, many of which are known to be harmful to the lung. Although far fewer marijuana than tobacco cigarettes are generally smoked on a daily basis, the pulmonary consequences of marijuana smoking may be magnified by the greater deposition of smoke particulates in the lung due to the differing manner in which marijuana is smoked. Whereas THC causes modest short-term bronchodilation, regular marijuana smoking produces a number of long-term pulmonary consequences, including chronic cough and sputum, histopathologic evidence of widespread airway inflammation and injury and immunohistochemical evidence of dysregulated growth of respiratory epithelial cells, that may be precursors to lung cancer. The THC in marijuana could contribute to some of these injurious changes through its ability to augment oxidative stress, cause mitochondrial dysfunction, and inhibit apoptosis. On the other hand, physiologic, clinical or epidemiologic evidence that marijuana smoking may lead to chronic obstructive pulmonary disease or respiratory cancer is limited and inconsistent. Habitual use of marijuana is also associated with abnormalities in the structure and function of alveolar macrophages, including impairment in microbial phagocytosis and killing that is associated with defective production of immunostimulatory cytokines and nitric oxide, thereby potentially predisposing to pulmonary infection. In view of the growing interest in medicinal marijuana, further epidemiologic studies are needed to clarify the true risks of regular marijuana smoking on respiratory health. Monaldi Arch Chest Dis 2005; 63: 2, 93-100.

Keywords: Marijuana, cannabis, respiratory symptoms, lung function, lung injury, alveolar macrophages, respiratory cancer.

Correspondence: Donald P. Tashkin, M.D.; Professor of Medicine; Division of Pulmonary \& Critical Care Medicine; Department of Medicine; David Geffen School of Medicine at UCLA; Los Angeles, CA 90095-1690; U.S.A.; e-mail dtashkin@mednet.ucla.edu

\section{Introduction}

A number of substances in addition to tobacco are inhaled for recreational use, including marijuana, crack cocaine, heroin, methamphetamine and phencyclidine. Of these, marijuana is the most commonly smoked substance, second only to tobacco. In the U.S., for example, among high school seniors, $21.2 \%$ admit to smoking marijuana within the previous month, compared to $24.4 \%$ for tobacco, while $6.0 \%$ state that they smoke marijuana daily, compared to $14.6 \%$ who smoke tobacco every day [1]. Marijuana smoke is composed of qualitatively the same ingredients that are present in tobacco smoke (e.g., carbon monoxide, cyanide, acrolein, benzene, vinyl chlorides, aldehydes, phenols, nitrosamines, reactive oxygen species, and a variety of polycyclic aromatic hydrocarbons [2, 3], with the major exceptions of the unique presence of $\Delta^{9}$-tetrahydrocannabinol (THC) and approximately 60 other cannabinoids only in marijuana [2, $3]$ and of nicotine only in tobacco. In view of this similarity in the chemical contents of the smoke from both substances, it might be expected that habitual inhalation of the smoke of marijuana might have injurious effects on the lung that are at least qualitatively similar to those resulting from regular tobacco smoking. Such tobacco-related pulmonary consequences are well-documented and include chronic obstructive pulmonary disease, bronchogenic carcinoma and an increased predisposition to lower respiratory tract infection. This report will review the evidence that addresses the question whether habitual smoking of marijuana can lead to long-term pulmonary effects similar to those of tobacco.

\section{Comparative acute effects of marijuana and tobacco on lung function}

Unlike tobacco, which causes modest, transient reflex-mediated bronchoconstriction, smoked marijuana causes acute bronchodilation both in healthy individuals [4] and asthmatic subjects [5] and rapidly reverses both exercise- and methacholine-induced bronchoconstriction in subjects with asthma [6]. The bronchodilator effect of marijuana is dependent on the concentration of $\Delta^{9}$ tetrahydrocannabinol (THC) and can be reproduced with oral THC in a dose-dependent manner [4]. The mechanism of THC-induced bronchodilation appears to be due to inhibition of acetylcholine release from efferent post-ganglionic nerve fibers that innervate airway smooth muscle, by analogy with the relaxant effect of $\mathrm{THC}$ on guinea pig ileum and inhibition by THC of salivary gland secretion in dogs [7-9]. Recently, cannabinoid type 1 (CB1) receptors (that mediate 
the neural effects of THC) were found on axon terminals of post-ganglionic parasympathetic nerve fibers in rat lung in close proximity to airway smooth muscle [10]. Stimulation of similar receptors in the guinea pig airway by the endogenous cannabinoid, anandamide, resulted in dose-dependent relaxation of capsaicin-contracted airway smooth muscle, suggesting that the endogenous cannabinoid system may play a regulatory role in the control of airway smooth muscle tone and providing an explanation for the bronchodilator effect of exogenous THC.

From a clinical perspective, on the other hand, smoking marijuana does not have a therapeutic role in obstructive airways diseases such as asthma since, despite its short-term bronchodilator properties, the long-term pulmonary consequences of marijuana smoking include several undesirable features, such as airway inflammation, edema and mucus hypersecretion, as described below.

\section{Long-term effects of habitual marijuana smoking}

\section{Effects on respiratory symptoms}

Respiratory symptoms were assessed using a standardized respiratory questionnaire [11] administered to a convenience sample of residents of Los Angeles, including 144 heavy, habitual smokers of marijuana only (MS), 135 regular smokers of both marijuana and tobacco (MTS), 97 control nonsmokers (NS) and 79 smokers of tobacco only (TS). On average, MS had smoked 3-4 marijuana cigarettes ("joints") per day for $>10$ years, while TS had smoked $>20$ tobacco cigarettes per day for $>10$ years. Of these subjects, $18-19 \%$ of MS admitted to chronic cough and sputum production, compared to $2-5 \%$ of NS, $24 \%$ of MS experienced wheeze on $\geq 21$ days a year compared to $7 \%$ of NS, and $13 \%$ of MS but only $2 \%$ of NS had $\geq 2$ episodes of acute bronchitis within the preceding 3 years [11]. The differences in occurrence of these respiratory symptoms between MS and NS was statistically significant $(p<0.05)$. While TS and MTS had a slightly higher prevalence than MS of similar symptoms, differences from MS were not statistically significant. These findings indicate that regular smoking of marijuana, even in the absence of tobacco, can lead to chronic bronchitis and a tendency to acute bronchitic episodes to an extent that is comparable to the effect of tobacco smoking; on the other hand, additive effects of marijuana and tobacco on chronic and acute respiratory symptoms were not apparent. Similar findings have been reported by other investigators in a randomly stratified population-based cohort in Tucson, Arizona [12] and a birth cohort in Dunedin, New Zealand [13], except that an additive effect of marijuana and tobacco was noted in the Arizona study [12].

\section{Effect on lung function}

Three groups of investigators have systematically examined lung function, both cross-sectional- ly and longitudinally, in well-characterized cohorts of habitual marijuana smokers and control nonsmokers of marijuana, controlling for the effects of tobacco [11-16]. In the Los Angeles cohort, most physiologic tests, including spirometry, as well as closing volume and density-dependence of flow indices (sensitive measures of small airways function) and diffusing capacity (a sensitive but nonspecific physiologic indicator of emphysema), were within normal limits and not different from the results in nonsmokers; the only exception was a slight but statistically significant increase in plethysmographically measured airway resistance in the MS, suggesting an impact of marijuana smoking on the large airways [11]. In addition, among MS, the annual rate of change in forced expired volume in $1 \mathrm{sec}\left(\mathrm{FEV}_{1}\right)$ measured over 8 years was not significantly different from that of NS [14]. In contrast, TS exhibited significant decrements compared to control NS in tests of small airways function cross-sectionally, as well as a significantly greater annual rate of decline in $\mathrm{FEV}_{1}$ than NS [14]. These findings suggest that heavy, habitual smoking of marijuana, in the absence of tobacco, does not produce the early or progressive physiologic changes that precede the clinical development of chronic obstructive pulmonary disease (COPD). These results are also consistent with findings in rats involving exposure to increasing concentrations of marijuana or tobacco smoke over one year that led to morphologic and physiologic changes of emphysema (decreased alveolar surface area and reduced lung elastic recoil) only in the tobacco-exposed rats but not in the animals exposed to a similar quantity of marijuana [17].

On the other hand, two other studies involving the cohorts from Tucson and Dunedin revealed evidence of mild airflow obstruction in association with marijuana use $[12,13]$. Moreover, in both of these cohorts the airflow obstruction progressed over time in the continuing marijuana users [15, 16]. In contrast to the Los Angeles study, therefore, these two reports suggest that regular use of marijuana may be a risk factor for the subsequent development of COPD. Further research is required to resolve these conflicting findings.

\section{Effects on visual evidence of airway injury and bronchial mucosal histopathology}

A subset of MS, MTS, TS and NS from the Los Angeles cohort underwent fiberoptic bronchoscopy, including videotaping of the tracheobronchial mucosa and/or performance of a series of mucosal biopsies [18]. The videotapes were blindly scored for the presence and degree of airway injury using a semiquantitative scoring system for mucosal erythema, swelling and increased secretions ("bronchitis index"). Visual evidence of airway injury of comparable degree was noted in both the MS and the TS with scores significantly higher than in control NS [18]. Mucosal biopsies from these same subjects revealed histopathologic abnormalities corresponding to the visual endoscopic changes of erythema, edema and increased 
secretions, including increased number and size of submucosal blood vessels, submucosal edema and hyperplasia of the mucus-secreting surface epithelial cells (goblet cells).

Light microscopy of bronchial mucosal biopsies in $40 \mathrm{MS}, 31 \mathrm{TS}, 44 \mathrm{MTS}$ and 53 NS [19] revealed extensive histopathologic alterations in the epithelium of the MS, including goblet cell hyperplasia, reserve cell hyperplasia, squamous metaplasia, cellular disorganization, nuclear atypia, increased mitotic index, increased nuclear/cytoplasmic ratio and inflammatory changes. The frequency of these abnormalities in the MS was comparable to that noted in the TS, with the suggestion of additive changes due to regular use of both substances in the MTS. Some of these histopathologic changes have been associated with the subsequent development of bronchogenic carcinoma in tobacco smokers [20].

Bronchial biopsies from 52 of these subjects were examined using immunohistology to detect abnormal expression of genes involved in the pathogenesis of lung cancer, including epidermal growth factor receptor (EGFR), increased expression of which can promote autonomous cell growth, and $\mathrm{Ki}-67$, a nuclear proliferation protein involved in cell replication [21]. Findings revealed striking overexpression of EGFR and Ki-67 in MS compared to NS, numerically even greater expression in MS than TS and the suggestion of additive effects in MTS. The light microscopic and immunohistochemical findings, when considered together, suggest that regular marijuana smoking injures the airway epithelium leading to dysregulation of bronchial epithelial cell growth and potentially to malignant transformation.

The similarity between the frequency, type and extent of the endoscopic and histopathologic evidence of injury in the large central airways of the smokers of marijuana alone and those of tobacco alone contrasts strikingly with the marked difference in the number of cigarettes smoked for the two types of substances (approximately 3-4 joints per day in the MS versus 22 tobacco cigarettes per day in the TS). This disparity could be due partly to the four-fold greater quantity of marijuana smoke particulates (tar) deposited in the respiratory tract compared to the deposition of tar generated from the same amount of smoked tobacco, thereby likely magnifying the impact of smoked marijuana on the lung. This difference in respiratory deposition of marijuana vs. tobacco tar has been attributed to differences in the way the two plant substances are smoked (e.g., four-fold longer breathholding time during marijuana than tobacco smoking) [22, 23]. A possible additional explanation for the similar impact of much smaller smoked quantities of daily marijuana than tobacco on respiratory symptoms and bronchial mucosal histopathology is that the THC and other cannabinoids unique to marijuana smoke might themselves have toxic effects that serve as an independent contributing factor to the changes observed in the large central airways. On the other hand, although possible structural changes in the distal lung could not be assessed bronchoscopically, results of the physiologic studies noted above suggest markedly different effects of tobacco and marijuana, i.e., evidence of small airways dysfunction and gas transfer abnormality in relation to tobacco and the absence of any similar abnormality in relation to marijuana. The reasons for these two different types of disparate effects of the two different plant substances on large airways vs. the distal lung are unclear but point to the possibility of contrasting effects of THC (and possibly other cannabinoids) on the biology of the epithelial cells in large vs. small airways that warrants further study.

\section{Effects on alveolar macrophages}

Alveolar macrophages (AM) are the primary immune cells residing in the distal air spaces of the lung and serve a critical role in host defense against infection, other noxious insults and malignancy. AM, recovered by bronchoalveolar lavage (BAL) at the time of bronchoscopy in participants in the Los Angeles study, were enumerated and examined for structural and functional changes [24]. Approximately 2, 3 and 4 times as many AM were recovered from MS, TS and MTS, respectively, than from NS, indicating an additive effect of marijuana and tobacco on AM accumulation [24]. The accumulation of AM in the lungs of MS probably represents an inflammatory response to noxious components, including oxyradicals, within the smoke, resulting in either increased AM recruitment to and/or increased replication in the lung. On ultrastructural examination, AM from MS as well as TS revealed large irregular-shaped cytoplasmic inclusions, most likely containing particulates taken up by these cells from the inhaled marijuana or tobacco tar; the number of these inclusions was dramatically increased in smokers of both marijuana and tobacco [25]. The presence of large numbers of abnormal inclusion bodies within the cytoplasm of AM from smokers of marijuana and/or tobacco might interfere with the function of these important immune effector cells.

Several aspects of the function of AM recovered from the aforementioned nonsmokers and smokers or marijuana and/or tobacco were evaluated: 1) phagocytic and killing activity against fungi and bacteria; 2) production of reactive oxygen and nitrogen intermediates during incubation with fungi or bacteria; 3) ability to produce proinflammatory cytokines when stimulated; and 4) cytotoxic activity against tumor cell targets. Significant findings from these studies of AM function may be summarized as follows:

- Impairment in fungicidal activity against Candida albicans and Candida tropicalis when AM from both MS and TS were compared to AM from control NS [26, 27];

- Impairment in phagocytosis and bactericidal activity against $S$. aureus by AM from MS but not TS [27];

- Reduction in basal superoxide production by AM from MS (contrasting with an increase in basal superoxide generation by AM from TS) [26]; 
- Impairment in generation of nitric oxide by AM from MS (but not TS), paralleling their impairment in bactericidal activity [28];

- Reduction in generation of proinflammatory cytokines, including TNF-alpha (TNF- $\alpha$ ) and granulocyte macrophage-colony stimulating factor (GM-CSF), by AM from MS when stimulated with bacterial lipopolysaccharide [27]; and

- Impairment in tumoricidal activity by AM from MS [27].

It is likely that THC, which is present only in the tar from marijuana smoke, is responsible for the functional differences noted between AM from MS vs. TS. One of the important clinical implications of the striking impairment in the function of AM from MS, particularly with regard to microbicidal activity, is that habitual smokers of marijuana may be at greater than normal risk for development of pneumonia. This possibility has been suggested by the results of a few epidemiologic studies (see below)

The defect in the bactericidal activity of AMs from MS appears to be due to THC-related impairment in production of nitric oxide, a reactive nitrogen intermediate that serves as an important effector molecule in bacterial killing. This hypothesis is supported by the finding that inhibition of nitric oxide synthase impairs killing of $S$. aureus by AMs from nonsmokers and tobacco smokers, but not by AMs from marijuana-only smokers [27]. More recent data have shown an inhibition of production of inducible nitric oxide synthase (iNOS) by AMs from marijuana smokers under conditions of the $S$. aureus killing assay that was restored by the addition of pro-inflammatory cytokines (e.g., INF $\gamma$ and GM-CSF) [28]; under the latter conditions, NO production and killing could be inhibited in the presence of an iNOS inhibitor, as shown with AMs from nonsmokers in bacterial culture $[27,28]$. These findings suggest that the impairment in the bactericidal activity of AMs from marijuana smokers might be due to marijuana-related inhibition of microbially-stimulated production of key pro-inflammatory cytokines that are needed, in turn, to induce iNOS. This hypothesis is further supported by other findings indicating that lipopolysaccharide-stimulated production of TNF- $\alpha$, IL- 6 and GM-CSF is inhibited by AMs from MS but not from TS [27].

\section{Clinical implications of marijuana-related impairment in alveolar macrophage function}

The clinical implications of the above findings are that regular marijuana smoking may compromise the lung's defense against infection by impairing the crucially important antimicrobial function of alveolar macrophages through inhibition of the production of pro-inflammatory cytokines that are required for immune activation. This possibility is supported by previous case reports of invasive Aspergillus pneumonia in immunocompromised patients with AIDS [29], chronic granulomatous disease [30], bone marrow transplantation [31], re- nal transplantation [32] or small cell lung cancer treated with chemotherapy [33], all of whom smoked marijuana. On the other hand, these cases of invasive pulmonary aspergillosis could be due primarily to the immune compromise from their underlying disease and/or the fact that marijuana is frequently contaminated with Aspergillus fumigatus [34], although a superimposed THC-related impairment of pulmonary host defenses could be an important additive factor predisposing to opportunistic infection. Marijuana has also been shown to be contaminated with potentially pathogenic gram-negative bacteria [35] that could similarly lead to pneumonia, especially in the setting of a compromised immune system.

As additional evidence of a link between regular marijuana use and infectious complications in immunocompromised patients, a few older epidemiologic studies have identified marijuana as a significant independent risk factor for the development of opportunistic pulmonary infection in HIV+ individuals [36-38]. In view of the increasing interest in medicinal marijuana, the possibility that marijuana smoking may predispose HIV+ patients to pneumonia requires further investigation by more rigorous epidemiologic studies.

\section{Does marijuana smoking predispose to respiratory cancer?}

Smoke contents of marijuana; cellular, tissue, and human studies

That marijuana smoking may be a risk factor for respiratory cancer is suggested by the chemical composition of marijuana smoke, as well as by evidence from cellular, tissue, animal and human studies.

First, marijuana smoke contains several of the same carcinogens and co-carcinogens as tobacco smoke, including vinyl chorides, phenols, nitrosamines, reactive oxygen species and various polycyclic aromatic hydrocarbons (PAHs) [3]. Furthermore, benzo[ $\alpha]$ pyrene, a highly procarcinogenic $\mathrm{PAH}$, is present in marijuana tar at a higher concentration than in tobacco tar [2,3].

Second, because of differences in the manner in which marijuana and tobacco are smoked, $\sim 4$-fold more of the tar from marijuana smoke than that from tobacco is deposited in the respiratory tract [22], thereby magnifying the level of exposure to carcinogens from each marijuana cigarette.

Third, hamster lung explants exposed to marijuana smoke for $\leq 2$ years developed abnormal cell growth and accelerated malignant transformation, changes that were even more prominent than those noted in explants exposed to tobacco [39].

Fourth, bronchial biopsies from heavy, habitual smokers of marijuana alone showed 1) light microscopic changes of epithelial hyperplasia, metaplasia and nuclear atypia that have been associated with the subsequent development of bronchogenic carcinoma in tobacco smokers $[19,20]$ and 2) immunohistochemical overexpression of Ki-67 (a proliferation marker), EGFR (epidermal growth 
factor receptor) and DNA ploidy (a marker of genetic instability), consistent with dysregulated growth and pre-tumor progression [21].

Fifth, in vitro exposure of endothelial cells (ECV 304 cell line), transformed lung cells (A549 cell line) and primary human airway epithelial cells to smoke from marijuana cigarettes stimulated the formation of more reactive oxygen species (ROS) than did exposure to the same amount of tobacco smoke and in direct proportion to the concentration of THC, indicating that marijuana smoke is a potent source of oxidative stress that could lead to cell injury, DNA damage and ultimately malignant transformation [40-44].

Sixth, exposure of pulmonary transformed A549 cells to tar extract from marijuana cigarettes or to pure THC is associated with a toxic effect on mitochondial electron transport, resulting in adenosine triphosphate (ATP) depletion [44]; the resulting mitochondrial function dysfunction could lead to inhibition of apoptosis, as has also been demonstrated following exposure of A549 cells to marijuana smoke extract [41], which, in turn, could play a role in carcinogenesis.

\section{Animal studies}

Non-small cell lung cancer cell lines implanted into immunocompetent mice displayed accelerated growth in the animals given THC intraperitoneally compared to control animals given vehicle alone [45]. Tissue assays from the tumors and spleens resected from THC-treated mice showed over-production of immunosuppressive cytokines (IL-10 and TGF- $\beta$ ) and underproduction of immunostimulatory cytokines (IL-2 and INF- $\gamma$ ) compared to control mice. The latter findings are consistent with a biasing effect of THC on T helper cell-type 1/T helper cell-type 2 (Th1/Th2) balance toward Th2 cells, as has been suggested by the results of other studies [46-48]. The augmentation of tumor cell growth by THC was blocked by co-administration of a selective antagonist against CB2 receptors (which are expressed primarily on immune cells). Thus, THC accelerates tumor growth in vivo by a cytokine-dependent and CB2 receptor-mediated mechanism that impairs the development of anti-tumor immunity.

\section{Clinical case series}

That marijuana may be a risk factor for respiratory cancer is further suggested by several small case series that reported an unusually high proportion of marijuana smokers among young individuals (< age 40-45 years) in whom upper aerodigestive tract cancers [49-52] or lung cancer [53] were diagnosed. However, case series do not provide strong evidence of a causal association since they are uncontrolled, indicating the need for well-designed epidemiologic studies.

\section{Epidemiological studies}

A few controlled epidemiological studies have addressed this issue with conflicting results. A large retrospectively analyzed cohort study of 65,000 subscribers to a Northern California health maintenance organization failed to find an elevated risk for tobacco-related malignancy, including lung and upper airway cancer, among ever or current marijuana smokers, after adjustment for tobacco smoking [54]. Weaknesses of this study included the relatively young age of the participants at the end of follow-up period and inclusion of few long-term or heavy marijuana smokers [54].

Two case-control studies that examined cannabis use as a possible risk factor for lung cancer were conducted in North Africa [55, 56]. A Tunisian study, including 110 lung cancer cases and 110 controls, reported a markedly elevated OR for ever use of cannabis $(\mathrm{OR}=8.2,95 \% \mathrm{CI}=1.3$ 15.5) [55]. Although cannabis is believed to be used in relatively large amounts in this region, unfortunately dose-response relationships were not assessed in this study. A second lung cancer casecontrol study, conducted in Northern Morocco, assessed the association between lung cancer and the use of hashish and kiff (a powdery preparation from the dried flowers of the female Cannabis sativa plant mixed with tobacco), with or without snuff [56]. Results of this study, which included 118 lung cancer cases and 235 controls, indicated that the combined use of hashish/kiff and snuff was associated with a 6.67 -fold greater risk [95\% C.I. 1.65-26.90] for developing lung cancer, while the risk was much lower for the use of hashish/kiff without snuff (1.93-fold [95\% C.I. 0.57-6.58]) and lower still for the use of snuff only ( $\mathrm{OR}=1.06$ [95\% CI $=0.33-3.47])$. Since kiff includes tobacco, the independent effect of cannabis cannot be assessed in this study.

Three case-control studies on marijuana smoking and risk of upper airway cancer have been published [57-59]. The first of these studies was a hospital-based U.S. study that included 173 head and neck cancer cases and 176 controls; the authors found that a history of daily or near-daily marijuana smoking was associated with a 2.6-fold greater risk [95\% C.I. 1.1-6.6] for developing head and neck cancer, after adjusting for other known risk factors, including tobacco smoking and alcohol use [57]. Furthermore, a dose-response relationship was found, and an even higher risk of marijuana smoking for the development of cancer was noted among younger individuals (< age 55 years). In contrast, a Washington State population-based case-control study of 407 cases of squamous cell cancer and carcinoma in situ of the oral cavity and 615 controls reported no association with marijuana use $(\mathrm{OR}=0.9$, C.I. 0.6-1.3) and no dose-response trends for frequency or duration of marijuana use [58]. A third head and neck cancer casecontrol study among young individuals $(\leq 45$ years) in the UK, including 116 cases of squamous cell cancer of the oral cavity and 207 matched controls, failed to find an association between cannabis use and oral cancer [59]. In the latter study, however, estimated risks associated with known major risk factors, namely tobacco and alcohol, were low, suggesting that the study may 
have lacked sensitivity for detecting marijuana as a potential risk factor for oral cancer.

Limitations of the above-cited epidemiologic studies include possible underreporting in countries where marijuana use is illegal, small sample size, sampling bias and failure to capture heavy or long-term marijuana users in the study population. Consequently, further well-designed, large-scale epidemiological studies that include a detailed assessment of marijuana exposure (frequency, duration and amount used) and that adjust adequately for tobacco smoking and other known risk factors are required to more definitively answer the question whether smoking of marijuana is or is not associated with an increased risk of respiratory cancer. The answer to this question is important for weighing the benefits and risks of medicinal marijuana use and clarifying the public health message regarding marijuana use.

\section{Pulmonary barotrauma and bullous lung disease}

A number of cases of spontaneous pneumothorax and/or pneumomediatinum associated with marijuana smoking have been reported [60-67]. The mechanism of this association is believed to be due to the repeated performance of prolonged Valsalva maneuvers during breathholding following deep inhalation of the smoke. These maneuvers could lead to barotrauma with rupture of subpleural blebs or alveoli and dissection of air around the vessels and bronchi to the pleural space or mediastinum. Alternatively, successive deep inhalations through a smoking apparatus with a high airflow resistance equivalent to Muller's maneuver could have a similar effect $[64,67]$ as a result of extreme negative intrathoracic pressure and high transmural pressure gradients inducing barotrauma.

Multiple large peripheral apical lung bullae have been observed in four young (27-46 years) male habitual marijuana smokers ( 2 joints to several pipefuls of marijuana per day), with little evidence on high resolution $\mathrm{CT}$ images of obvious parenchymal disease elsewhere in the lung [68]. Significant airflow obstruction was present in one of these cases and moderate reductions in gas transfer in two. A similarly uncommon form of bullous lung disease has been noted in young male tobacco smokers [69]. However, three of the four cases had smoked relatively little tobacco (3-7 pack-years or 2-5 cigars/day), suggesting that their habitual marijuana smoking may have played a role in the pathogenesis of their bullous lung disease.

\section{Conclusions}

The smoke from marijuana, the second most commonly smoked substance after tobacco, contains, in addition to THC, a large number of toxic gases and particulates (including high concentrations of procarcinogenic polycyclic aromatic hydrocarbons) that are capable of causing lung injury and potentially respiratory malignancy. Whereas
THC produces short-term bronchodilation by relaxing airway smooth muscle, heavy habitual use of marijuana is associated with a number of adverse pulmonary consequences (table 1). These include 1) symptoms of acute and chronic bronchitis, 2) conflicting findings concerning the presence or absence of mild, progressive airflow obstruction, 3) endoscopic and microscopic evidence of airway wall edema, vascular congestion and increased mucous secretion, 4) extensive histopathologic and immunohistochemical evidence of damage and dysregulated growth of the tracheobronchial epithelium, and 5) accumulation of increased numbers of alveolar macrophages that demonstrate impaired antimicrobial and tumoricidal function and impaired ability to generate immunostimulatory cytokines and inducible nitric oxide synthase and nitric oxide, an important effector molecule in microbial killing. These features raise concern that marijuana smoking may be a risk factor 1) for opportunistic infection, especially in already immunocompromised patients due to AIDS, organ transplanatation or cancer chemotherapy, and 2) for upper and lower respiratory tract cancer. Additional, large-scale epidemiologic studies are required to address these concerns.

Table 1. - Pulmonary Consequences of Habitual Marijuana Use

- Increased prevalence of acute and chronic bronchitis [11-13]

- Conflicting evidence for and against mild, progressive airflow obstruction [11-16]

- Visual endoscopic evidence of airway injury (mucosal erythema, edema and increased secretions) [18]

- Microscopic alterations in tracheobronchial epithelium and subepithelium (squamous metaplasia, reserve cell hyperplasia, goblet cell hyperplasia, loss of ciliated epithelium, basement membrane thickening, epithelial inflammation, cellular disorganization, increased nuclear to cytoplasmic ratio) [19]

- Overexpression of molecular markers of pre-tumor progression (epidermal growth factor receptor, Ki-67) in tracheobronchial epithelial cells [21]

- Increased numbers of alveolar macrophages recovered by bronchoalveolar lavage [24]

- Impairment in alveolar macrophage microbicidal and tumoricidal activity [26, 27]

- Impairment in stimulated alveolar macrophage production of proinflammatory cytokines [27] and expression of inducible nitric oxide synthase [28]

- Older epidemiologic evidence of an association between marijuana smoking and the development of opportunistic pulmonary infection in HIV+ individuals [36-38]

- Conflicting epidemiologic evidence for $[55,56]$ or against [54] an association of marijuana use with lung cancer

- Conflicting epidemiologic evidence for [57] or against $[58,59]$ an association of marijuana use with head and neck cancer 
Acknowledgment: This work is supported in part by a grant from the National Institutes of Health/National Institute on Drug Abuse no. R37 DA03018.

\section{References}

1. Johnston LD, O’Malley PM, Bachman JG. Monitoring the Future. National Survey Results on Drug Use, 19752003, Vol. I and II, National Institute on Drug Abuse. USDHHS NIH Publication No. 04-5507, 2004.

2. Hoffmann D, Brunneman DK, Gori GB et al. On the carcinogenicity of marijuana smoke. Recent Adv Phytochem 1975; 9: 63-81.

3. Novotny M, Merli F, Weisler D et al. Fractionation and capillary gas chromatographic mass spectrometric characterization of the neutral components in marijuana and tobacco smoke concentrates. J Chromatogr 1982; 238: 141-150.

4. Tashkin DP, Shapiro BJ, Frank IM. Acute pulmonary physiologic effects of smoked marijuana and oral $\Delta^{9}$ tetrahydrocannabinol in healthy young men. $N$ Engl $J$ Med 1973; 289: 336-341.

5. Tashkin DP, Shapiro BJ, Frank IM. Acute effects of smoked marijuana and oral $\Delta^{9}$-tetrahydrocannabinol on specific airway conductance in asthmatic subjects. Am Rev Respir Dis 1974; 109: 420-428,

6. Tashkin DP, Shapiro BJ, Lee EY. Effects of smoked marijuana in experimentally induced asthma. Am Rev Respir Dis 1975; 112: 377-386,

7. Layman JM, Milton AS. Some actions of delta-1 tetrahydrocannabinol and cannabidiol at cholinergic junctions. Br J Pharmacol 1971; 41: 379P-380P.

8. Coutts AA, Pertwee RG. Inhibition by cannabinoid receptor agonists of acetylcholine release from guinea-pig myenteric plexus. Br J Pharmacol 1997; 121: 15571566.

9. Carvero I, Buckley JP, Jandhyala BS. Parasympatholytic activity of (-)- $\Delta^{9}$-trans-tetrahydrocannabinol in mongrel dogs. Eur J Pharmacol 1972; 19: 301-304.

10. Calignano A, Katona I, Desarnaud F et al. Bidirectional control of airway responsiveness by endogenous cannabinoids. Nature 2000; 408: 96-101.

11. Tashkin DP, Coulson AH, Clark VA et al. Respiratory symptoms and lung function in habitual, heavy smokers of marijuana alone, smokers of marijuana and tobacco, smokers of tobacco alone, and nonsmokers. Am Rev Respir Dis 1987; 135: 209-216.

12. Bloom JW, Kaltenborn WT, Paoletti P et al. Respiratory effects of non-tobacco cigarettes. Brit Med J 1987; 295: 1516-1518.

13. Taylor DR, Poulton R, Moffitt TE et al. The respiratory effects of cannabis dependence in young adults. $\mathrm{Ad}$ diction 2000; 95: 1669-1677.

14. Tashkin DP, Simmons MS, Sherrill D et al. Heavy habitual marijuana smoking does not cause an accelerated decline in $\mathrm{FEV}_{1}$ with age: a longitudinal study. Am J Respir Crit Care Med 1997; 155: 141-148.

15. Sherrill DL, Krzyzanowski M, Bloom JW et al. Respiratory effects of non-tobacco cigarettes: A longitudinal study in general population. Internat J Epidem 1991; 20: 132-137.

16. Taylor DR., Fergusson DM, Milne BJ et al. A longitudinal study of the effects of tobacco and cannabis exposure on lung function in young adults. Addiction 2002; 97: 1055-1061.

17. Huber GL, Mahajan VK. The comparative response of the lung to marihuana or tobacco smoke inhalation. In: G. Chesher, P. Consroe \& R. Musty, eds Marijuana: An International Research Report. Proceedings of Melbourne Symposium on Cannabis 2-4 September, 1987 (National Campaign Against Drug Abuse Monograph
Series No. 7). Canberra, Australia: Australian Government Publishing Service, 1987: 19-24.

18. Roth MD, Arora A, Barsky SH et al. Visual and pathologic evidence of injury to the airways of young marijuana smokers. Am J Respir Crit Care Med 1998; 157: 928-937.

19. Fligiel SEG, Roth MD, Kleerup EC et al. Tracheobronchial histopathology in habitual smokers of cocaine, marijuana and/or tobacco. Chest 1997; 112: 319326.

20. Auerbach O, Stout AP, Hammond EC et al. Changes in bronchial epithelium in relation to sex, age, residence, smoking and pneumonia. $N$ Engl J Med 1962; 267: 111119.

21. Barsky SH, Roth MD, Kleerup EC et al. Similar molecular alterations in bronchial epithellium are observed in habitual smokers of marijuana, cocaine and/or tobacco. J National Cancer Institute 1998; 90: 1198-1204.

22. Wu T-C, Tashkin DP, Djahed B et al. Pulmonary hazards of smoking marijuana as compared with tobacco. N Engl J Med 1988; 318: 347-351.

23. Tashkin DP, Gliederer F, Rose $\mathrm{J}$ et al. Effects of varying marijuana smoking profile on deposition of tar and absorption of CO and delta-9-THC. Pharmacology, Biochemistry and Behavior 1991; 40: 651-656.

24. Barbers RG, Gong H Jr, Tashkin DP et al. Differential examination of bronchoalveolar lavage cells in tobacco cigarette and marijuana smokers. Am Rev Respir Dis 1987; 135: 1271-1275.

25. Beals TF, Fligiel SEG, Stuth $\mathrm{S}$ et al. Morphological alterations of alveolar macrophages from marijuana smokers. Am Rev Respir Dis 139 (part 2): A336, 1989.

26. Sherman MP, Campbell LA, Gong H Jr et al. Respiratory burst and microbicidal characteristics of pulmonary alveolar macrophages recovered from smokers of marijuana alone, smokers of tobacco alone, smokers of marijuana and tobacco and nonsmokers. Am Rev Respir Dis 1991; 144: 1351-1356.

27. Baldwin GC, Tashkin DP, Buckley DM et al. Habitual smoking of marijuana and cocaine impairs alveolar macrophage function and cytokine production. $J$ Respir Crit Care Med 1997; 156: 1606-1613.

28. Shay AH, Choi R, Whittaker K et al. Nitric oxide: A mediator of alveolar macrophage antimicrobial activity compromised in cocaine and marijuana smokers. $J$ Infect Dis 2003; 187: 700-4.

29. Denning DW, Follansbee SE, Scolaro M et al. Pulmonary aspergillosis in the acquired immunodeficiency syndrome. N Engl J Med 1991; 324: 654-662.

30. Chusid MJ, Gelfland JA, Nutter C et al. Pulmonary aspergillosis, inhalation of contaminated marijuana smoke, chronic granulomatous disease (letter). Ann Intern Med 1975; 82: 682-683.

31. Hamadeh R, Ardehali A, Locksley RM et al. Fatal aspergillosis associated with smoking contaminated marijuana in a marrow transplant recipient. Chest 1988; 94 : 432-433

32. Marks WH, Florence L, Lieberman J et al. Successfully treated invasive pulmonary aspergillosis associated with smoking marijuana in a renal transplant recipient. Transplantation 1996; 61: 1771-1783.

33. Sutton S, Lum BL, Torti FM. Possible risk of invasive pulmonary aspergillosis with marijuana use during chemotherapy for small cell lung cancer. Drug Intell Clin Pharm 1986; 20: 289-291.

34. Kagen SL, Kurup VP, Sohnle PC et al. Marijuana smoking and fungal sensitization. J Allergy Clin Immunol 1983; 71: 389-393.

35. Ungerleider JT, Andrysiak T, Tashkin DP et al. Contamination of marihuana cigarettes with pathogenic bacteria - possible source of infection in cancer patients. Cancer Treatment Reports 1982; 66: 589-591. 
36. Newell GR, Mansell PW, Wilson MB et al. Risk factor analysis among men referred for possible acquired immune deficiency syndrome. Preventive Medicine 1985; 14: 81-91.

37. Tindall B, Philpot CR, Cooper DA et al. The Sydney AIDS project: Development of acquired immunodeficiency syndrome in a goup of HIV seropositive homosexual men. Aust NZ J Med 1988; 18: 8-15.

38. Caiaffa WT, Vlahov D, Graham NM et al. Drug smoking, Pneumocystis carinii pneumonia, and immunosuppresion increase risk of bacterial pneumonia in human immunodeficiency virus-seropositive infection drug users. Am Rev Respir Dis 1994; 150: 1493-98.

39. Leuchtenberger C, Leuchtenberger R. Cytological and cytochemical studies of the effects of fresh marihuana cigarette smoke on growth and DNA memtabolism of a nimal and human lung cultures. In: Braude MC \& Szara S, eds The Pharmacology of Marijuana New York: Raven Press, 1976: 595-612.

40. Sarafian TA, Marques JA, Shau H et al. Oxidative stress produced by cannabinoids in marijuana smoke. Am J Respir Mol Cell Biol 1999; 20: 1286-1293.

41. Sarafian TA, Roth MD, Tashkin DP. Marijuana smoked and $\Delta^{9}$-tetrahydrocannabinol promote necrotic cell injury but inhibit fas-mediated apoptosis. Toxicology and Applied Pharmacology 2001; 174: 264-72.

42. Sarafian TA, Kouyoumjian S, Tashkin D et al. Synergistic cytotoxicity of $\Delta^{9}$-tetrahydrocannabinol and butylated hydroxyanisole. Toxciology Letters 2002; 133: 171-179.

43. Roth MD, Baldwin GC, Tashkin DP. Effects of delta-9tetrahydrocannabinol on human immune function and host defense. Chem. Phys. Lipids. Special Issue: Cannabinoids: Chemistry and Biology 2002; 121: 229239.

44. Sarafian TA, Louyoumjian S, Khoshaghideh $\mathrm{F}$ et al. Tetrahydrocannabinol disrupts mitochondrial function and cell energetics. Am J Physiol Lung Cell Mol Physiol 2003; L298-L306.

45. Zhu LX, Sharma S, Stolina M, Gardner B et al. $\Delta^{9-}$ tetrahydrocannabinol inhibits antitumor immune-surveillance by a CB2 receptor-mediated, cytokine-dependent pathway. J Immunol 2000; 165: 373-380.

46. Yuan M, Kiertscher SM, Cheng Q et al. $\Delta^{9}$-tetrahydrocannabinol regulates Th1/Th2 cytokine balance in activated human T cells. J Neuroimmunol 2002; 133: 124131.

47. Newton CA, Klein TW, Friedman H. Secondary immunity to Legionella pneumophila and Th1 activity are suppressed by $\Delta^{9}$-tetrahydrocannabinol injection. Infection and Immun 1994; 62: 4015-20

48. Klein TW, Friedman H, Specter S. Marijuana, immunity and infection. J Neuroimmunol 1998; 83: 102-115, 1998.

49. Taylor FM. Marijuana as a potential respiratory tract carcinogen: A retrospective analysis of a community hospital population. South Med J 1988; 81: 1213-1216.

50. Donald PJ. Advanced malignancy in the young marijuana smoker. Advances Exper Med Biol 1991; 288: 33-56.

51. Endicott JN, Skipper P, Hernandez L. Marijuana and head and neck cancer. In: Friedman H, ed Drugs of Abuse, Immunity and AIDS. New York, NY: Plenum Press, 1993: 107-113.

52. Fung M, Gallagher C, Machtay M. Lung and aero-digestive cancers in young marijuana smokers. Tumori 1999; 85: 140-142.

53. Sridhar KS, Raub WA, Weatherby NL et al. Possible role of marijuana smoking as a carcinogen in the development of lung cancer at a young age. J Psychoactive Drugs 1994; 26: 285-88.

54. Sidney S, Beck JE, Tekawa IS et al. Marijuana use and cancer incidence. Am J Public Health 1997; 585-590.

55. Hsairi M, Achour N, Zouari B et al. [Etiologic factors in primary bronchial carcinoma in Tunisia.] Tunis Med. 1993; 71: 265-8.

56. Sasco AJ, Merill RM, Dari I et al. A case-control study of lung cancer in Casablanca, Morocco. Cancer Causes \& Control 2002; 13: 609-16.

57. Zhang ZF, Morgenstern H, Spitz MR et al. Marijuana use and increased risk of squamous cell carcinoma of the head and neck. Cancer Epidemiol Biomarkers Prev 1999; 8: 1071-8.

58. Rosenblatt KA, Daling JR, Chen C et al. Marijuana use and risk of oral squamous cell carcinoma. Cancer Res. 2004; 64: 4049-54.

59. Llewellyn CD, Linklater K, Bell J et al. An analysis of risk factors for oral cancer in young people: a case-control study. Oral Oncol 2004; 40: 304-13.

60. Feldman AL, Sullivan JT, Passero MA et al. Pneumothorax in polysubstance abusing marijuana and tobacco smokers: 3 cases. J Substance Abuse 1993; 5: 183-6.

61. Mattox KL. Pneumomediastinum in heroin and marijuana users. JACEP 1976; 5: 26-8.

62. Miller WE, Spiekerman RE, Hepper NG. Pneumomediastinum resulting from performing Valsalva maneuvers during marijuana smoking. Chest 1972; 62: 233-4.

63. Goodyear K, Laws D, Turner J. Bilateral spontaneous pneumothorax in a cannabis smoker. J R Soc Med 2004; 97: 435-6.

64. Herrejon SA, Blanquer OJ, Simo MM et al. [Pneumothorax due to drug inhalation.] An Med Interna 1992; 9: 137-9.

65. Luque MA III, Cavallaro DL, Torres M et al. Pneumomediastinum, pneumothorax, and subcutaneous emphysema after alternate cocaine inhalation and marijuana smoking. Pediatr Emerg Care 1987; 3: 107-9.

66. Birrer RB, Calderon J. Pneumothorax, pneumomediastinum, and pneumopericardium following Valsalva's maneuver during marijuana smoking. NY State J Med 1984; 84: 619-20.

67. Hazouard E, Koninck JC, Attucci S et al. Pneumorachis and pneumomediastinum caused by repeated Muller's maneuvers: complications of marijuana smoking. Ann Emerg Med 2001; 38: 694-7.

68. Johnson MK, Smith RP, Morrison D et al. Large lung bullae in marijuana smokers. Thorax 2000; 55: 340-2.

69. Stern EJ, Webb WR, Weinacker A et al. Idiopathic giant bullous emphysema (vanishing lung syndrome): imaging findings in nine patients. AJR 1994; 162: 279-82. 